https://helda.helsinki.fi

\title{
Frequency comb generation in a continuous-wave pumped second-order nonlinear waveguide resonator
}

\section{Abdallah, Zeina}

IEEE

2019

Abdallah , Z , Stefszky , M , Ulvila , V , Silberhorn , C \& Vainio , M 2019 , Frequency comb generation in a continuous-wave pumped second-order nonlinear waveguide resonator . in 2019 CONFERENCE ON LASERS AND ELECTRO-OPTICS (CLEO) . , 8750403 , Conference on Lasers and Electro-Optics, IEEE, Conference on Lasers and Electro-Optics , San Jose , California , United States , 05/05/2019 . https://doi.org/10.1364/CLEO_SI.2019.SM3O.4

http://hdl.handle.net/10138/311060

https://doi.org/10.1364/CLEO_SI.2019.SM3O.4

unspecified

acceptedVersion

Downloaded from Helda, University of Helsinki institutional repository.

This is an electronic reprint of the original article.

This reprint may differ from the original in pagination and typographic detail.

Please cite the original version. 


\title{
Frequency comb generation in a continuous-wave pumped second-order nonlinear waveguide resonator
}

\author{
Zeina Abdallah ${ }^{1}$, Michael Stefszky ${ }^{2,3}$, Ville Ulvila ${ }^{3,4}$, Christine Silberhorn ${ }^{2}$, and Markku Vainio ${ }^{1,4}$. \\ ${ }^{1}$ Laboratory of Photonics, Korkeakoulunkatu 3, Tampere University of Technology, FI-33720, Tampere, Finland \\ ${ }^{2}$ Integrated Quantum Optics, Applied Physics, Paderborn University, Warburger Strasse 100, 33098 Paderborn, Germany \\ ${ }^{3}$ Molecular Science, Department of Chemistry, P.O. Box 55 (A.I. Virtasen aukio 1), FI-00014 University of Helsinki, Finland \\ ${ }^{4}$ VTT Technical Research Centre of Finland Ltd, Vuorimiehentie 3, 02150 Espoo, Finland \\ zeina.abdallah@tut.fi
}

\begin{abstract}
An optical frequency comb has been experimentally demonstrated using an integrated system based on a lithium niobate waveguide resonator featuring a strong quadratic nonlinearity. Our theoretical model shows good agreement with the experimental results. (C) 2019 The Author(s). OCIS codes: 070.5753 Resonators, 190.0190 Nonlinear optics.
\end{abstract}

An optical frequency comb (OFC) generator is a source of laser light whose spectrum consists of a series of precisely spaced sharp spectral spikes. These generators are used on a large scale for many applications, ranging from high precision metrology to biomedical/environmental spectroscopy. To date, the state-of-the-art method for OFC generation has been mode locked lasers. Aiming towards miniaturization, broadband frequency combs have also been demonstrated by means of continuous-wave (CW) pumped nonlinear microresonators, which utilize the third-order cubic optical nonlinearity, known as the optical Kerr effect. While being a very promising technology, Kerr combs face some challenges, especially those pertaining to the properties of the microresonator material. The cubic nonlinearity, which is often described by the nonlinear refractive index, is typically small in the available optical materials. Consequently, Kerr comb generation requires high laser intensity, and so a microresonator with a very high quality $(\mathrm{Q})$ factor is needed.

A new approach for miniaturized $\mathrm{CW}$ pumped comb generators is based on cascaded quadratic nonlinear (CQN) processes, which are fundamentally much stronger than the cubic nonlinear ones [1]. Hence, CQNs offer a path to highly efficient OFC generation, which alleviates the requirement for high resonator $\mathrm{Q}$ factor and, thus potentially simplifies, and allows for some strategy to mitigate the unwanted thermal effects when the power is so high. Interestingly, it is demonstrated here that these type of comb sources could be altered to an integrated version $[3,4]$. This integrated system is based on a well-developed titanium indiffused lithium niobate waveguide resonator (Ti:PPLN) that features a strong quadratic nonlinearity [4].

By pumping a cavity-enhanced second-harmonic generation (SHG) with a $\mathrm{CW}$ field $\left(\omega_{p}\right)$, the combs generated around the fundamental field $\left(\omega_{p}\right)$ and the second-harmonic field $\left(2 \omega_{p}\right)$ can be explained by the presence of a consecutive application of two nonlinear operators; the generated second-harmonic power, initially converted from the fundamental wave, acts as a pump for the reverse (down-conversion) process.

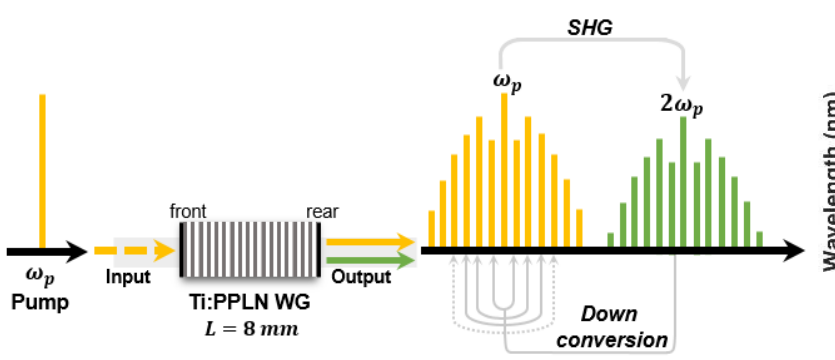

(a)

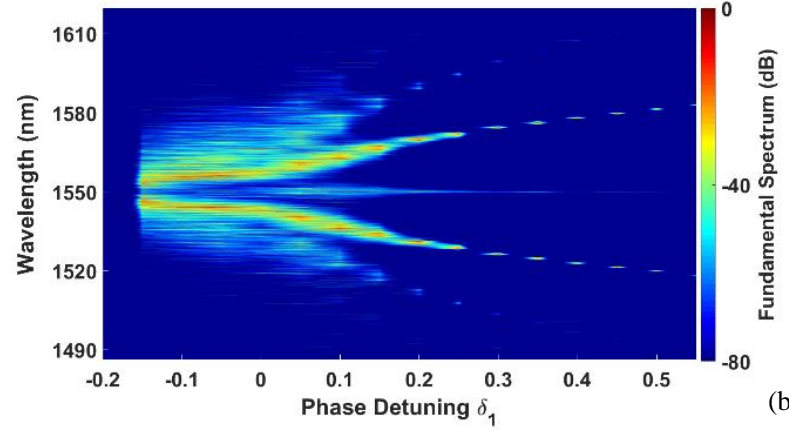

(b)

Fig. 1 (a) Schematic of the CW pumped integrated OFC generation in a quadratic nonlinear cavity. By pumping this waveguide by a strong CW laser at $\omega_{p}$, an optical frequency comb around $\omega_{p}$ and $2 \omega_{p}$ are generated. (b) Spectral dynamics around the fundamental frequency as the cavity detuning $\delta_{1}$ is swept across a resonance with a $330 \mathrm{~mW}$ pump power. The spectrum is normalized to the maximum power in the field.

From the theoretical and modeling point of view, there are several ways to explain the comb generation in a CQN system. A new study reveals that the modulation instability (MI) is fundamentally reinforced by the temporal walk-off between the interacting fields, and consequently, triggers the formation of frequency combs and drifting temporal patterns [5]. The related predictive model successfully simulates the combs generated in both singly and doubly 
resonant systems. However, further expansion and improvement of this model were required in order to efficiently simulate and predict the behavior of this specific integrated system, since only the pump field resonates inside the cavity while the second-harmonic field exits the waveguide at the rear facet (as shown in Fig.1 (a)).

The (full map) coupled equations were numerically solved using the split-step fourth-order Runge-Kutta method. Figure 1 (b) shows the simulated intracavity spectrum dynamic around the fundamental frequency as the detuning $\left(\delta_{1}\right)$ is increased. From this figure, one can determine the detuning range where the MI emerges, and this range was around -0.15 and 0.55 . In fact, when the system transits from a stable to MI pattern (around $\delta_{1}=-0.15$ ), the spectrum is composed of spectral lines spaced by a multiple FSRs. As $\delta_{1}$ increases, the sidebands are frequency shifted farther away from the pump.

The fundamental field exiting the rear facet of the resonator is measured using an optical spectrum analyzer. The measurements were taken as the pump laser wavelength was scanned from red to blue over the cavity resonance. Figure 2 (a-c) represent some measured envelope spectra for different cavity detuning. As the pump wavelength approaches the cavity resonance, the optical comb generated becomes stronger. These MI peaks equidistant at around $5 \mathrm{~nm}$ from the fundamental wavelength and, reach a maximum magnitude of about $-10 \mathrm{dBc}$.
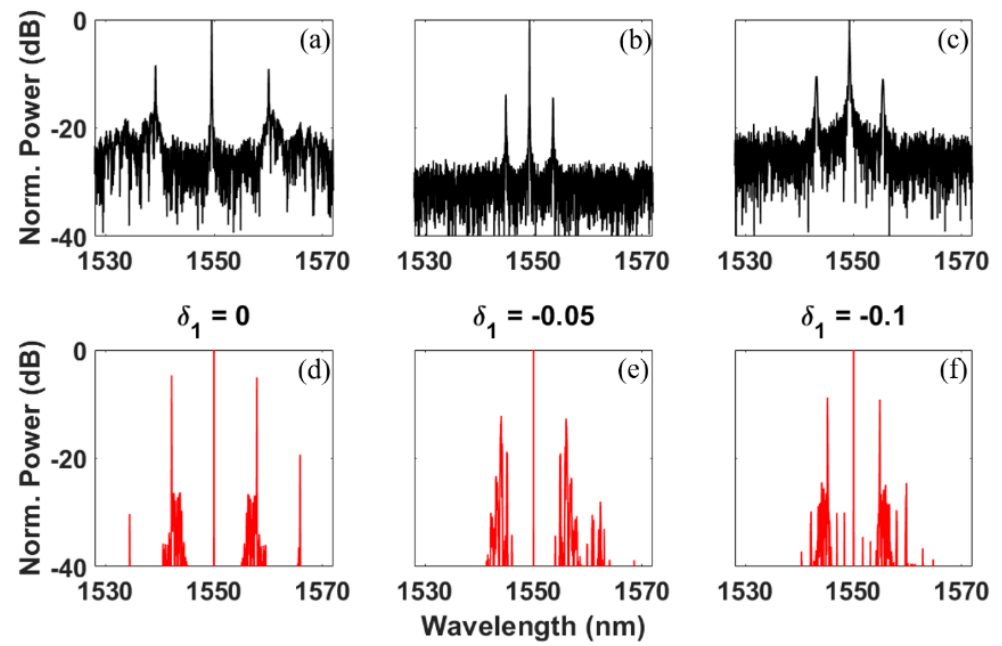

Fig. 2 (a)-(c) Measured optical spectra for $330 \mathrm{~mW}$ pump power as the pump laser is scanned from red to blue. (d)-(f) Predicted fundamental spectra for $330 \mathrm{~mW}$ pump power for different detuning values.

Then, we compare the measured spectrum with the theoretically predicted ones (Fig. 2 (d-f)). Close to the cavity resonance, the theoretical model shows a good agreement with the experimental results. It has to be noted that the thermal effect and the photorefraction may have been limiting factors during the experiments, and these effects are not included in the existing models.

We have demonstrated experimentally and theoretically optical comb envelope generation using a CW pumped integrated titanium indiffused resonator. This integrated device results in a more mechanically robust miniaturized system, where the field is highly confined with a high nonlinear interaction strength, leading to low threshold powers. However, more work has to be done and several questions need to be addressed toward a clear understanding of the underlying physics of the CQN frequency comb. In addition, the influence of various mechanisms on the stability of the system of high practical importance, such as thermal effects and laser noise, should also be included in the models. Therefore, it is of high importance to quantitatively investigate and describe these effects.

\section{References}

[1] V. Ulvila, C. R. Philips, L. Halonen and M. Vainio, "Frequency comb generation by a continuous-wave-pumped optical parametric oscillator based on cascading quadratic nonlinearities," Opt. Lett. 38, 4281 (2013).

[2] M. Stefszky, V. Ulvila, Z. Abdallah, C. Silberhorn, and M. Vainio, "Towards optical-frequency-comb generation in continuous-wave-pumped titanium-indiffused lithium-niobate waveguide resonators,” Phys. Rev. A 98, 053850 (2018).

[3] R. Ikuta, M. Asano, T. Tani, T. Yamamoto, and N. Imoto,"Frequency comb generation in a quadratic nonlinear waveguide resonator," Opt. Lett. 26, 15551 (2018).

[4] M. Stefszky, R. Ricken, C. Eigner, V. Quiring, H. Herrmann and C. Silberhorn, “A high-power waveguide resonator second harmonic device with external conversion efficiency up to 75\%," J. Opt. 20, 065501 (2018).

[5] F. Leo, T. Hansson, I. Ricciardi, M. De Rosa, S. Wabnitz and M. Erkintalo, "Walk-off-Induced Modulation Instability, Temporal Pattern Formation, and Frequency Comb Generation in Cavity-Enhanced Second-Harmonic Generation,” PRL 116, 033901 (2016). 